\title{
Surgical treatment of congenital thoracolumbar spondyloptosis in a 2-year-old child with vertebral column resection and posterior-only circumferential reconstruction of the spine column: case report
}

\author{
Loyola V. Gressot, MD, Javier A. Mata, MD, Thomas G. Luerssen, MD, and Andrew Jea, MD \\ Division of Pediatric Neurosurgery, Texas Children's Hospital, Department of Neurosurgery, Baylor College of Medicine, \\ Houston, Texas
}

\begin{abstract}
Spondyloptosis refers to complete dislocation of a vertebral body onto another. The L5-S1 level is frequently affected. As this condition is rare, few published reports describing its clinical features and surgical outcomes exist, especially in the pediatric patient population.

The authors report the presentation, pathological findings, and radiographic studies of a 2-year-old girl who presented to Texas Children's Hospital with a history since birth of progressive spastic paraparesis. Preoperative CT and MRI showed severe spinal cord compression associated with T11-12 spondyloptosis. The patient underwent a single-stage posterior approach for complete resection of the dysplastic vertebral bodies at the apex of the spinal deformity with reconstruction and stabilization of the vertebral column using a titanium expandable cage and pedicle screws. At the 12-month follow-up, the patient remained neurologically stable without any radiographic evidence of instrumentation failure or loss of alignment.

To the best of the authors' knowledge, there have been only 2 other children with congenital thoracolumbar spondyloptosis treated with the above-described strategy. The authors describe their case and review the literature to discuss the aggregate clinical features, surgical strategies, and operative outcomes for congenital thoracolumbar spondyloptosis.

http://thejns.org/doi/abs/10.3171/2014.9.PEDS14151
\end{abstract}

KEY WORDS spondyloptosis; vertebral column resection; congenital; pediatric; spine

$\mathrm{C}$ ONGENITAL spondyloptosis was first reported by Dubousset in 1973 as a severe form of congenital kyphosis. ${ }^{8}$ The poor natural history and potential for neurological devastation due to congenital kyphosis has been well described. ${ }^{28}$ Congenital kyphosis is characterized by an anterior defect in formation. The pathognomonic radiographic sign is the abrupt displacement of a vertebral body at a single level in the sagittal plane. This abrupt angulation of the spinal canal is associated with deformation of the spinal cord.

Patients with various types of vertebral column malformations of the thoracolumbar spine have been grouped together using terms such as "congenital spondyloptosis," "congenital vertebral dislocation," "congenital dislocated spine," "segmental spinal dysgenesis," "medial spinal aplasia," "congenital spinal stenosis," "congenital duplica- tion of the spinal canal," "congenital kyphosis and subluxation of the thoracolumbar spine due to vertebral aplasia," and "congenital lumbar kypholisthesis." "3,5,8,10,12,16,20,21,23,25-29 It has been suggested that all of these terms describe a single fundamental malformation. ${ }^{5,12}$ This inconsistent nomenclature has led to the misclassification of patients.

Relatively few published reports regarding the surgical treatment of congenital thoracolumbar spondyloptosis exist in the literature..$^{26,27,29}$ The general recommendation is to perform a circumferential reconstruction and fusion of the vertebral column by utilizing both anterior strut grafting and posterior fusion. Posterior decompression alone for spinal cord decompression remains controversial, as mixed neurological outcomes have been reported using this technique. ${ }^{4,7,30}$ It should always be performed in conjunction with fusion. We describe a case involving a single-stage 
posterior-only approach for circumferential neurological decompression, vertebral column resection, deformity reduction, anterior expandable titanium cage placement, and posterior instrumented fusion in a 2-year-old child with symptomatic congenital thoracolumbar spondyloptosis. To the best of our knowledge, only 2 previously published cases have been treated using this strategy.

\section{Case Report}

\section{History and Examination}

This 2-year-old girl presented to the Neuro-Spine Clinic at Texas Children's Hospital with progressive spastic paraparesis and a neurogenic bladder, both of which she had had since birth. The patient had evidence of atrophy, hyperreflexia, and spasticity in both legs, as well as clubfoot deformities. She was unable to voluntarily move her legs and was wheelchair bound. Examination showed an obvious bony gibbous in her lower back, but she was without other cutaneous stigmata of spinal dysraphism.

Computed tomography scanning of the spine showed T11-12 and T12-L1 spondyloptosis, T11-12 dysmorphic facet joint subluxation, bilateral dislocated facet joints at the T12-L2 articulations, agenesis of the posterior elements T-11 through L-1, a hypoplastic T-12 body, and dysplastic L-1 with severe kyphotic deformity (Fig. 1A). Magnetic resonance imaging of the spine demonstrated severe thoracolumbar dysgenesis with canal compromise as well as severe hypoplasia of the spinal cord rostral to the bony anomaly and low-lying conus medullaris with thickened filum terminale (Fig. 1B). Skeletal survey and renal ultrasound did not demonstrate any additional anomalies other than clubfeet.

\section{Operation}

Surgery was planned to stabilize the spine and decom- press the spinal cord. After inducing general anesthesia, needle electrodes were placed in the upper and lower extremities to monitor somatosensory evoked potentials and motor evoked potentials. Baseline reliable evoked potentials were unattainable in the lower extremities. The patient was then positioned prone, and an S-shaped incision centered over the bony gibbous was made. The incision was planned to allow greater lateral access than the standard midline approach as a modification of the lateral extracavitary approach, and thus allowing manipulation of the thecal sac without undue traction on the spinal cord. Subperiosteal dissection was then performed to expose the posterior spine, taking special care at dysplastic levels with known laminar defects. Laminectomies were performed from T-8 to L-4, and pedicle screws were placed under direct palpation at T-8, T-9, T-10, L-2, L-3, and L-4 using cervical spine instrumentation (Vertex, Medtronic Sofamor Danek). Fluoroscopy was used to confirm screw placement, and a temporary rod was inserted.

The T-12 nerve roots were ligated and sectioned to allow greater manipulation of the thecal sac without excessive spinal cord traction. From the posterior approach, a vertebral column resection was performed to remove the entirety of the dysplastic vertebrae at T-11, T-12, and L-1. The deformity was then reduced, and a $13 \times 16-\mathrm{mm}$ expandable cage was placed from T-10 to L-2 (Fig. 2). Final rods were placed, the exposed bony surfaces were decorticated, and morselized autograft with bone morphogenetic protein (BMP; Infuse, Medtronic Sofamor Danek) was used as graft material (Fig. 3). The filum was not untethered during this operation, as the patient had a clinical presentation consistent with spinal cord compression at the level of deformity, not with signs or symptoms of tethered cord. Prophylactic untethering of the cord was not justified given the added risk of morbidity, such as CSF leakage in the large dead space of the dissection.
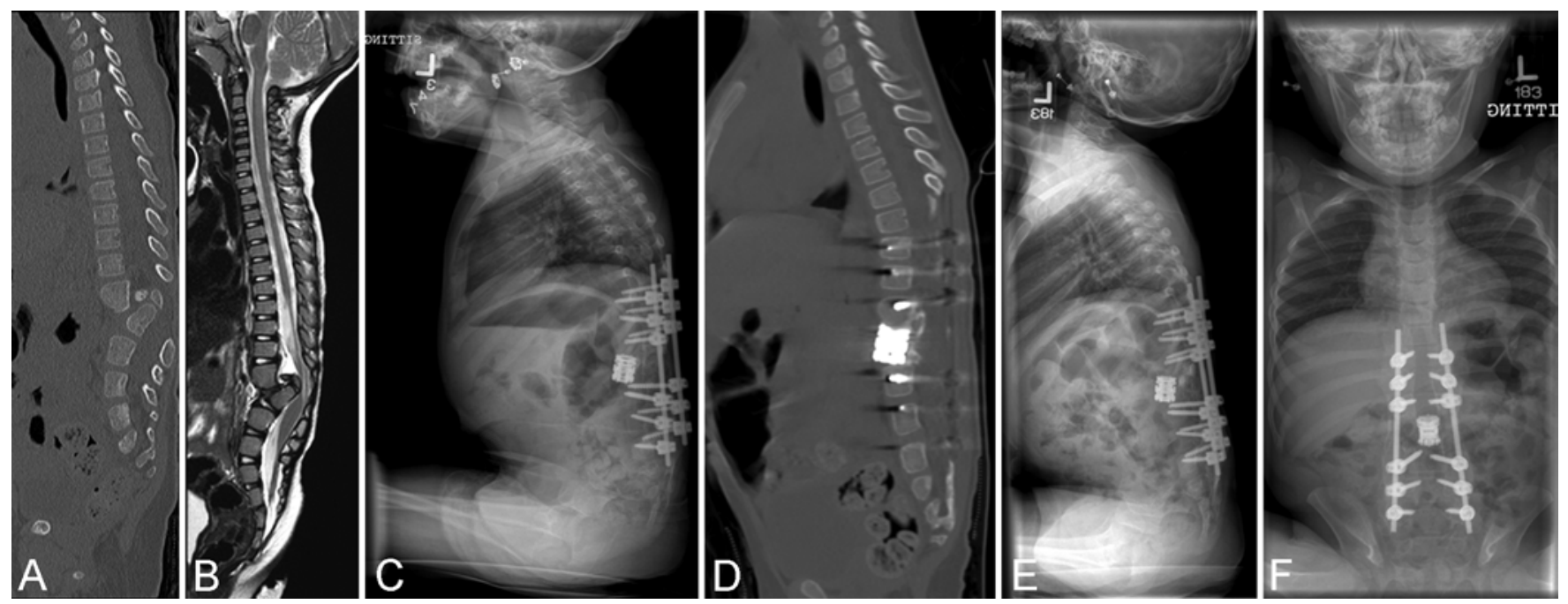

FIG. 1. Preoperative spine CT (A) showed T11-12 and T12-L1 spondyloptosis as well as spina bifida occulta of T11-L1, a hypoplastic T-12 body, and dysplastic L-1 with gibbous deformity. Thoracic MRI. Preoperative sagittal T2-weighted MR image (B) demonstrates severe spinal cord compression and buckling of the spinal column at the T11-12 level. Sagittal (C) and parasagittal (D) CTs without contrast obtained at 3 months after surgery, showing complete resection of the dysplastic T-11, T-12, and L-1 vertebral bodies and stabilization of the vertebral column with an expandable titanium cage and pedicle screws from T-8 to L-4. Bony fusion is visible behind the cage and in the posterolateral zones. Lateral $(E)$ and anteroposterior $(F)$ upright radiographs obtained at 12 months after surgery, showing maintenance of alignment without evidence of hardware failure. 


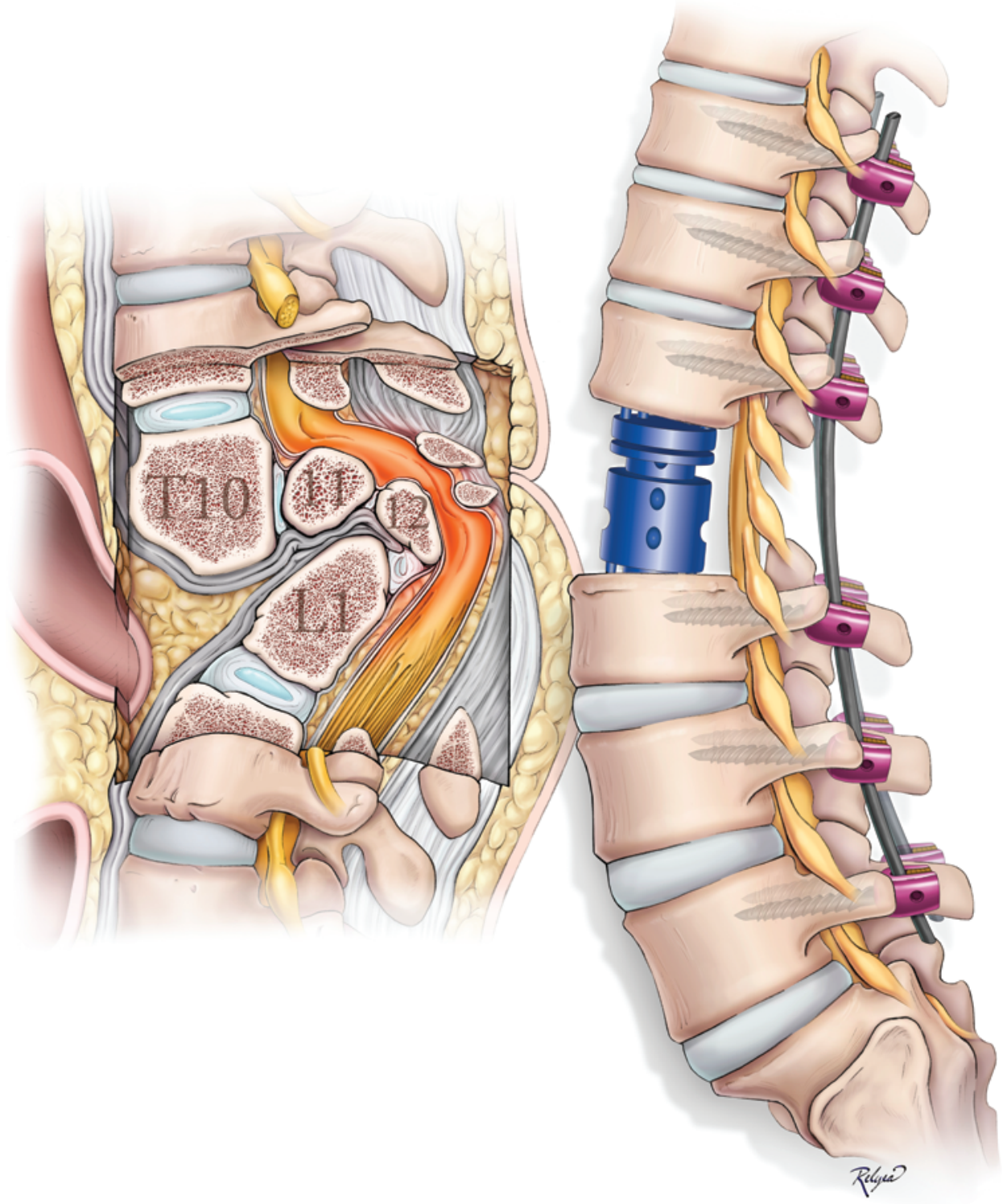

FIG. 2. Illustration showing spondyloptosis deformity (left) and final reduction with spinal instrumentation in place (right). Copyright Katherine Relyea. Published with permission.

\section{Postoperative Course}

There were no changes in the neurological function of the patient after surgery. She remained densely paraparetic in the lower extremities. Postoperative upright radiographs and CT scans showed satisfactory hardware placement and spinal alignment. The patient was discharged home on postoperative Day 5. Computed tomography scanning performed at 3 months after surgery demonstrated evidence of bony fusion behind the cage and on either side of the midline (Fig. 1C and D). At 12 months after surgery, the patient remained clinically and radiographically stable with no loss of alignment or evidence of instrumentation failure on whole-spine radiographs (Fig. 1E and F). Her motor function in the lower extremities was unchanged, but there was improved bladder emptying as documented on formal urodynamics.

\section{Discussion}

\section{Classification and Nomenclature}

The classification of this deformity remains controversial. $5,26,29,30$ The deformity is characterized by abrupt displacement of the entire spine at a single level, leading to instability and the potential for neurological deterioration..$^{5,10}$ The term "congenital spondyloptosis" carries this meaning of complete dislocation of the vertebral column. ${ }^{1}$ We advocate using this terminology as the designation for this disorder.

\section{Embryology}

The embryonic mechanism responsible for this resultant spinal column dislocation remains poorly understood. ${ }^{5}$ Vertebral column development begins in the 4 th 


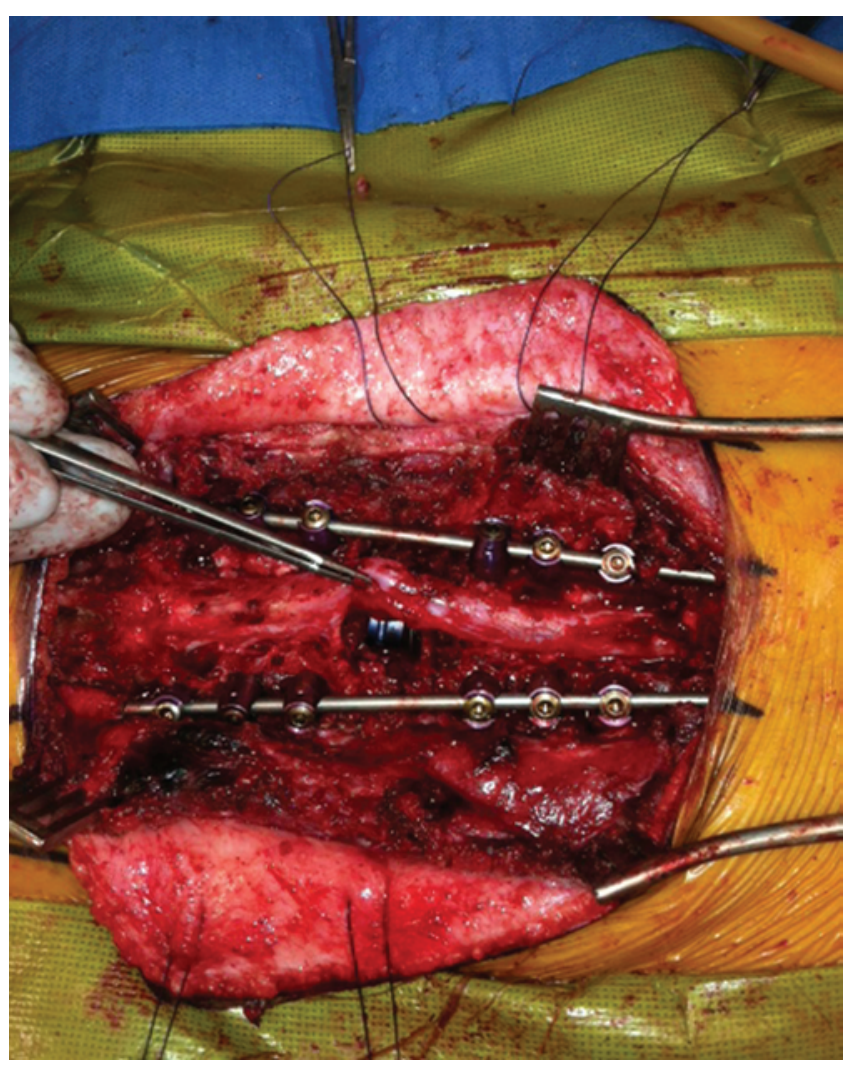

FIG. 3. Intraoperative photograph demonstrates thecal sac with the T-12 nerve roots divided, pedicle screws and rods in place, and an expandable cage from $T-10$ to $L-2$.

week of embryonic development, arising from the sclerotome of somites, which form from paraxial mesoderm, and continues through the 6th week when chondrification of the vertebra begins..$^{5,17,22}$ Dias et al. have proposed that this anomaly results from a "buckling" of the embryonic spine during development Weeks 4-6, with segmental translation at a single level of the notochord, adjacent somites, and neural tube, the three central neuraxial structures. ${ }^{5}$ They argue that this mechanism would explain the relative preservation of the major neuraxial structures at each level as well as the relatively normal and intact spinal cord and column superior and inferior to the defect. ${ }^{5}$ Congenital spondyloptosis typically occurs in isolation without associated anomalies, suggesting deformation of the developing spine as opposed to malformation. ${ }^{5}$ The forces causing this buckling remain unknown. Dias et al. proposed a mechanical force as a probable explanation. ${ }^{5}$ Congenital spondyloptosis may be seen at the transition point of the thoracolumbar junction where the spine changes curvature from kyphosis to lordosis; the ribs stabilize the upper thoracic spine and the diaphragm attaches to the upper lumbar vertebrae, creating a point vulnerable to mechanical deformation at the thoracolumbar junction. ${ }^{5}$ Alternate explanations may include a defect in somatogenesis, possibly due to defective homeobox genes or other developmental genes active in embryogenesis., ${ }^{5,17}$

\section{Literature Review}

We performed PubMed and OVID searches using the key phrases "congenital spondyloptosis" and "congenital vertebral dislocation." Inclusion criteria sought papers from peer-reviewed journals that described the surgical treatment of congenital thoracolumbar (T10-L2) spondyloptosis in pediatric patients (age $<18$ years). ${ }^{23,25-27,29}$ References in these articles were further analyzed for other papers not captured by the initial literature search. Papers were excluded if the surgical approach, arthrodesis type, or surgical outcome was not detailed., $3,510,20,21,30$ Thus, in addition to our case, we obtained and reviewed from the literature the data for 23 patients with surgically treated congenital thoracolumbar spondyloptosis.

The mean age in our aggregate series of 24 patients (Table 1) was 10.9 months (range $0-72$ months) at presentation and 43.3 months (range 1-204 months) at the time of surgery. There were 14 boys and 10 girls. The most common clinical findings on presentation were congenital paraparesis/paraplegia (8 patients), deformity without neurological deficit (10 patients), and progressive myelopathy, paraplegia after minor trauma, and hyperreflexia (1 patient each). The most frequent spinal levels involved at the thoracolumbar junction were T-10 (1 patient), T-11 (6 patients), T-12 (9 patients), L-1 (13 patients), and L-2 (4 patients).

Surgical treatment included anterior-only decompression and arthrodesis in 1 patient (Case 7); posterior-only decompression and arthrodesis in 2 patients (Cases 13 and 19); and combined anterior and posterior decompression and fusion in 21 patients. Viehweger et al. ${ }^{27}$ described single-stage vertebrectomy plus anterior and posterior fusion in 2 patients. However, there were subtle differences between these cases and our case. Those authors used a fibular strut graft and laminar hooks that were later removed; we used an anterior expandable cage and more rigid pedicle screw fixation.

Two patients experienced neurological deterioration after surgery, although it was transient in one. Three patients improved after surgery, and 17 were neurologically stable in follow-up. The patients were followed up for a mean of 75.3 months (range 9-215 months).

\section{Treatment}

The rare occurrence of congenital spondyloptosis combined with the inconsistencies in terminology for classifying the disorder makes elucidating its ideal treatment difficult. A wide range of therapeutic options from observation to circumferential fusion has been described. Several authors have reported the development of progressive neurological deficits in children with congenital spondyloptosis, suggesting a poor natural history with the risk of progressive deformity and the development of myelopathy. ${ }^{5,29} \mathrm{In}$ our aggregate series, $3(12.5 \%)$ of 24 patients with congenital thoracolumbar spondyloptosis deteriorated neurologically prior to surgery.

Surgical stabilization in infants is complicated given their small size, concerns regarding long-term growth potential, less ossified bone, and difficulty in achieving appropriate immobilization..$^{15}$ The interval of time between age at presentation and age at surgery was 28.6 months (range 0-132 months). This delay may have been an attempt by the treating surgeon to operate at a time when the 
旁恶

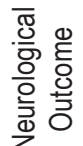

ఏ

융

䒕

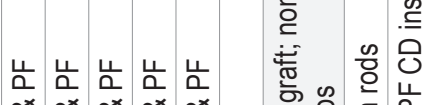

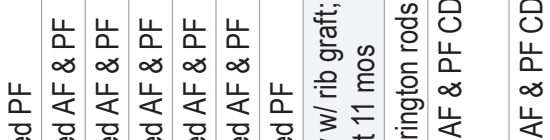

क्ष

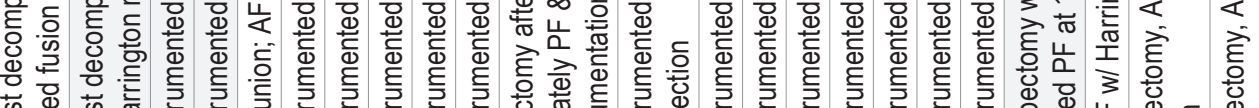

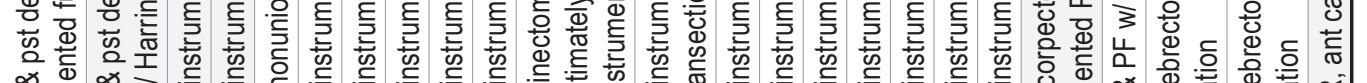

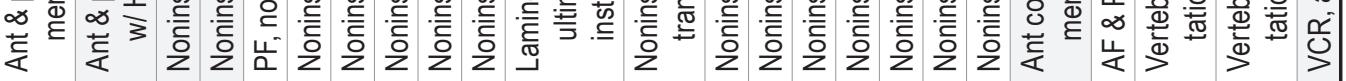

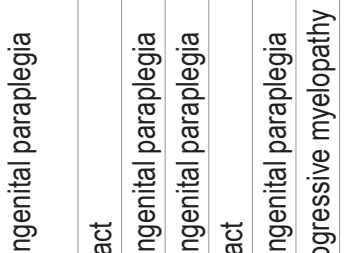

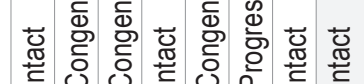

ठ

$\sum \sum \Sigma \Sigma \Sigma u \Sigma u$ u

$\stackrel{\circ}{\check{E}}$

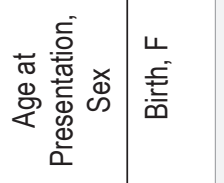

ญ

i -

$\sim$

u $\sum \sum$

焉

$m+\operatorname{monab}=$

ঙำ

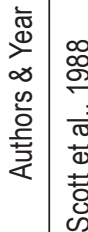

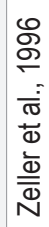

$\simeq$

$m$ 느는

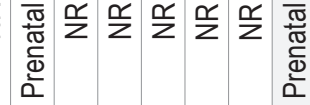

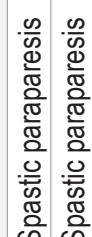

吕

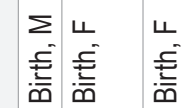

중 
skeleton of an older child is more mature and when there is greater potential to immobilize the spine with rigid instrumentation. Many authors recommend a combined anterior and posterior fusion in two stages if necessary. 5,26,27,29 This was accomplished in 21 of the children (87.5\%) in our aggregate series. Posterior fusion alone, with or without decompression, may be inadequate given the highly unstable nature of this disorder, and supplementation of the fusion from an anterior approach is usually required.

Whether decompression in addition to fusion is beneficial is a matter of controversy. The compressive pathology is ventral to the spinal cord; dorsal decompression would seem to have little effect on neurological compression. Moreover, neurological deterioration in most cases is attributable to spinal instability, and posterior decompression has the potential to worsen spinal stability. Therefore, upfront spinal fusion is regarded as the most important surgical treatment. 5,26,27,29 Shapiro and Herring have commented that there may be a role for decompression in patients with acute-onset myelopathy provided that sufficient stability can be achieved by arthrodesis. ${ }^{26}$

Given the above rationale, we found surgery to be indicated in our patient to halt the progression of kyphotic deformity, preserve normal biomechanics, and ameliorate neurological symptoms. Modern techniques, such as vertebral column resection (VCR) via a posterior approach, allow for circumferential decompression of the spinal cord, correction of acute segmental deformity, and resection of the bony gibbous. In our patient, VCR was followed by vertebral column reconstruction with an anterior expandable titanium cage and posterior instrumentation 3 levels above and 3 levels below the VCR. This resulted in complete decompression of the spinal cord and reduction and stabilization of the kyphotic deformity. To the best of our knowledge, while spinal instrumentation has been used in the treatment of congenital thoracolumbar spondyloptosis in very young children, ${ }^{26,27,29}$ our patient represents one of the few children treated with a single-stage posterior-only VCR and circumferential vertebral column reconstruction with anterior expandable titanium cage and posterior rigid pedicle screw fixation. We hope that the combination of anterior and posterior decompression with spinal instrumentation and the restoration of more physiological biomechanics of the spine will increase stability and be associated with a long-term favorable outcome, as documented in the literature. ${ }^{5,26,27,29}$ At the last follow-up in our patient, the motor examination and sagittal spinal alignment were stable with some improvement in bladder function. However, she will need to be followed up for the next several years until the age of skeletal maturity to monitor for the development of a progressive kyphotic or crankshaft deformity.

\section{Long-Term Consequences of Fusion in a Growing Spine}

A major consideration in pediatric spine fusion is the fact that the pediatric spine will continue to grow throughout childhood and adolescence. Possible complications include restricted range of motion, development of secondary deformity, and loss of growth potential. Fusions have often been avoided in children and adolescents for this reason. Any surgical approach must take into account the skeletal immaturity of young children and should minimize the number of fused segments, especially in the thoracic spine, to avoid complications, such as iatrogenic short trunk, crankshaft deformity, and pulmonary hypoplasia from restricted growth of the rib cage.

Growth of the anterior column after a posterior column fusion may cause a "crankshaft phenomenon" in which the anterior vertebral bodies continue to grow anteriorly disproportionate to the posterior elements in which all longitudinal growth has ceased..$^{9,14,19}$ This results in pivoting of the vertebral bodies on the posterior fusion causing progressive rotation and angulation of the spine. This problem occurs more commonly in skeletally immature patients younger than 10 years of age and in patients with idiopathic as opposed to congenital scoliosis. ${ }^{19}$ The relatively low incidence of crankshaft phenomenon in congenital scoliosis may be caused by the "sick" anterior growth plate encountered in this population as opposed to the "healthy" anterior growth plate seen in patients with idiopathic scoliosis

The addition of anterior fusion has been proposed as a possible solution to crankshaft phenomena. ${ }^{6,18}$ Various authors have proposed age, riser sign of 0 , peak height velocity, and open triradiate cartilage as factors to assess patients at risk. ${ }^{24}$ Other authors question the necessity and efficacy of using anterior fusion to prevent a crankshaft phenomena. ${ }^{2}$ Some authors believe that the stiffer segmental fixation of newer instrumentation systems (for example, Cotrel-Dubousset, Texas Scottish Rite Hospital, and Isola instrumentation) may prevent this secondary deformity better than the older Harrington, Luque, or Harri-Luque instrumentation. This is currently an important and active area of research interest.

We also supplemented the fusion construct with recombinant human BMP-2. There are concerns regarding the routine and "off-label" substitution or supplementation of autologous or allograft bone graft with recombinant human BMP-2. The most significant concerns involve the possibility of bony overgrowth, interaction with exposed dura mater, cancer risk, systemic toxicity, local toxicity, immunogenicity, osteoclastic activation, and effects on distal organs..$^{11,13}$

\section{Conclusions}

The avoidance of neurological morbidity requires early diagnosis and stabilization. Posterior spinal decompression and fusion alone do not seem to be sufficient to achieve neurological improvement nor a solid fusion in this type of congenital anomaly. We recommend a single-stage posterior-only approach for circumferential decompression and fusion. This surgical strategy seems to be well tolerated by patients in the pediatric age group. However, long-term follow-up is necessary to determine the deleterious iatrogenic effects of this surgical approach, including short trunk and crankshaft deformity.

\section{References}

1. Alicioglu B, Demir MK, Durmus Y: Congenital upper thoracic spondyloptosis with multiple other associated anomalies. Pediatr Int 51:848-850, 2009 
2. Burton DC, Asher MA, Lai SM: Scoliosis correction maintenance in skeletally immature patients with idiopathic scoliosis. Is anterior fusion really necessary? Spine (Phila Pa 1976) 25:61-68, 2000

3. Carstens C, Schneider E, Lang R: Medial spinal aplasia-a rare manifestation of congenital paraplegia: report of six cases and review of the literature. J Pediatr Orthop 12:485489, 1992

4. Chou SN: The treatment of paralysis associated with kyphosis: role of anterior decompression. Clin Orthop Relat Res (128):149-154, 1977

5. Dias MS, Li V, Landi M, Schwend R, Grabb P: The embryogenesis of congenital vertebral dislocation: early embryonic buckling? Pediatr Neurosurg 29:281-289, 1998

6. Dohin B, Dubousset JF: Prevention of the crankshaft phenomenon with anterior spinal epiphysiodesis in surgical treatment of severe scoliosis of the younger patient. Eur Spine J 3:165-168, 1994

7. Dubousset J: Congenital kyphosis and lordosis, in Weinstein SL (ed): The Pediatric Spine: Principles and Practice. New York: Raven Press, 1994, Vol 1, pp 245-258

8. Dubousset J, Duval-Beaupère G, Anquez L: Déformations vertébrales congénitales compliquées de troubles neurologiques, in Rougerie J (ed): Les Compressions Médullaires Non Traumatiques de l'Enfant. Paris: Masson, 1973, pp 193-207

9. Dubousset J, Herring JA, Shufflebarger H: The crankshaft phenomenon. J Pediatr Orthop 9:541-550, 1989

10. Faciszewski T, Winter RB, Lonstein JE, Sane S, Erickson D: Segmental spinal dysgenesis. A disorder different from spinal agenesis. J Bone Joint Surg Am 77:530-537, 1995

11. Fahim DK, Whitehead WE, Curry DJ, Dauser RC, Luerssen TG, Jea A: Routine use of recombinant human bone morphogenetic protein-2 in posterior fusions of the pediatric spine: safety profile and efficacy in the early postoperative period. Neurosurgery 67:1195-1204, 2010

12. Flynn JM, Otsuka NY, Emans JB, Hall JE, Hresko MT: Segmental spinal dysgenesis: early neurologic deterioration and treatment. J Pediatr Orthop 17:100-104, 1997

13. Gressot LV, Patel AJ, Hwang SW, Fulkerson DH, Jea A: RhBMP-2 for L5-S1 arthrodesis in long fusions to the pelvis for neuromuscular spinal deformity in the pediatric age group: analysis of 11 patients. Childs Nerv Syst 30:249-255, 2014

14. Hefti FL, McMaster MJ: The effect of the adolescent growth spurt on early posterior spinal fusion in infantile and juvenile idiopathic scoliosis. J Bone Joint Surg Br 65:247-254, 1983

15. Hwang SW, Gressot LV, Chern JJ, Relyea K, Jea A: Complications of occipital screw placement for occipitocervical fusion in children. J Neurosurg Pediatr 9:586-593, 2012

16. Jo DJ, Seo EM, Kim KT, Kim SM, Lee SH: Lumbosacral spondyloptosis treated using partial reduction and pedicular transvertebral screw fixation in an osteoporotic elderly patient. J Neurosurg Spine 16:206-209, 2012

17. Keynes RJ, Stern CD: Mechanisms of vertebrate segmentation. Development 103:413-429, 1988
18. Lapinksy AS, Richards BS: Preventing the crankshaft phenomenon by combining anterior fusion with posterior instrumentation. Does it work? Spine (Phila Pa 1976) 20:13921398,1995

19. Lee CS, Nachemson AL: The crankshaft phenomenon after posterior Harrington fusion in skeletally immature patients with thoracic or thoracolumbar idiopathic scoliosis followed to maturity. Spine (Phila Pa 1976) 22:58-67, 1997

20. Lorenzo RL, Hungerford GD, Blumenthal BI, Bradford BF, Sanchez F, Haranath BS: Congenital kyphosis and subluxation of the thoraco-lumbar spine due to vertebral aplasia. Skeletal Radiol 10:255-257, 1983

21. McKay DW, Nason SS: Congenital duplication of the spinal canal. A case report. Spine (Phila Pa 1976) 5:390-391, 1980

22. O'Rahilly R, Meyer DB: The timing and sequence of events in the development of the human vertebral column during the embryonic period proper. Anat Embryol (Berl) 157:167-176, 1979

23. Philips MF, Dormans J, Drummond D, Schut L, Sutton LN: Progressive congenital kyphosis: report of five cases and review of the literature. Pediatr Neurosurg 26:130-143, 1997

24. Sanders JO, Little DG, Richards BS: Prediction of the crankshaft phenomenon by peak height velocity. Spine (Phila Pa 1976) 22:1352-1357, 1997

25. Scott RM, Wolpert SM, Bartoshesky LE, Zimbler S, Karlin L: Segmental spinal dysgenesis. Neurosurgery 22:739-744, 1988

26. Shapiro J, Herring J: Congenital vertebral displacement. J Bone Joint Surg Am 75:656-662, 1993

27. Viehweger E, Giacomelli MC, Glard Y, Launay F, Petit P, Jouve JL, et al: Congenital dislocated spine: implications for orthopaedic management. J Pediatr Orthop 29:362-368, 2009

28. Winter RB: Congenital kyphosis. Clin Orthop Relat Res (128):26-32, 1977

29. Zeller RD, Ghanem I, Dubousset J: The congenital dislocated spine. Spine (Phila Pa 1976) 21:1235-1240, 1996

30. Zidorn T, Krauspe R, Eulert J: Dorsal hemivertebrae in children's lumbar spines. Spine (Phila Pa 1976) 19:2456-2460, 1994

\section{Author Contributions}

Conception and design: Jea. Acquisition of data: Jea, Mata. Analysis and interpretation of data: Jea, Mata. Drafting the article: Jea, Gressot. Critically revising the article: Jea, Gressot. Reviewed submitted version of manuscript: Jea, Gressot, Mata. Approved the final version of the manuscript on behalf of all authors: Jea. Study supervision: Luerssen.

\section{Correspondence}

Andrew Jea, Texas Children's Hospital, 6621 Fannin St., CCC 1230.01, 12th Fl., Houston, TX 77030. email: ahjea@ texaschildrens.org. 